\title{
Transmit Power Control for Wireless Body Area Networks using Novel Channel Prediction
}

\author{
David B. Smith ${ }^{\dagger}$, Leif W. Hanlen ${ }^{\dagger}$, Dino Miniutti ${ }^{\dagger}$ \\ National ICT Australia (NICTA) \\ (e-mail: \{David.Smith, Leif.Hanlen, Dino.Miniutti\}@nicta.com.au)
}

\begin{abstract}
We present a predictor for real Body-Area-Network (BAN) channels that is accurate for up to 2 seconds, even with a nominal channel coherence time of $500 \mathrm{~ms}$. The predictor utilizes the partial-periodicity of measured BAN channels using the previous 4 seconds of channel gain values. We demonstrate use of this predictor for power control with open-access and private channel measurements. When used under a realistic setting for IEEE 802.15.6, with packet loss less than $10 \%$, we show that the accurate channel predictor does not translate into substantial reduction in packet loss or power usage over a simple sampleand-hold method, even though it is a more accurate predictor than sample-and-hold.
\end{abstract}

\section{INTRODUCTION}

The first major application of wireless networks of sensors around the body, body-area-networks (BANs) [1], is likely to be healthcare. In consideration of the technical requirements for BAN [2] and the use in health-care: long-term, reliable, low-power communication is vitally important. Adaptive power control is a possible solution that can facilitate longterm low-power operation, and also improve reliability.

The basis of the work here is large sets of empirical BAN data collected using small wearable radios, with ten adult human subjects engaged in every-day activity, generally over periods of two hours or more. Such comprehensive dynamic data for the BAN channel facilitates good overall characterization $^{1}$. This data also enables the testing and development of schemes that can be used to enhance BAN communications, such as the power control scheme in [4], where a very simple method of effective power control using the prediction from the last held sample was presented ${ }^{2}$.

Here the data was further analysed to answer the following:

1) Is it possible to improve long-term channel prediction over that of the held sample in the BAN channel?

2) Can this predictor be used to provide an adaptive power control method that reduces transmit circuit power consumption and improves reliability?

${ }^{\dagger}$ D. Smith, L. Hanlen and D. Miniutti hold adjunct appointments with the Australian National University.

${ }^{\ddagger}$ National ICT Australia is funded by the Australian Government as represented by the Department of Broadband, Communications and the Digital Economy and the Australian Research Council through the ICT Centre of Excellence program.

${ }^{1}$ First and Second order statistics for this data can be found in [3].

${ }^{2} \mathrm{~A}$ more complex power control method, evaluated using in-body to onbody simulated data, is presented in [5]
3) With likely periods for beacons, superframes, and intervals between packets for a given hub to sensor link, does this adaptive power control still work?

4) Is an enhancement of the power control method using a short-term mean threshold effective?

We demonstrate that effective long-term BAN channel prediction, up to $2 \mathrm{~s}$ ahead, is possible, and that it improves on the method of simply using the last sample held as a prediction. Partial periodicity of the fading channel is used by applying a non-traditional least squares method to weight a held sample by a previously received contiguous portion of channel gain samples. With direct power allocation based on the prediction, there are significant improvements in terms of normalized mean-square power error and bias for all BAN receive sensitivities likely at $2.4 \mathrm{GHz}$. Adaptive power control using the predictor can both reduce transmit power consumption and improve reliability. Similar to the method for a held sample in [4], adapting power allocation based on a shortterm mean path loss shows good performance, and works given typical BAN superframe periods and intervals between packets for a given hub-to-sensor link.

A description of the experimental method follows in the next section. Section III provides description and analysis of the predictor, provides an algorithm adapting the predictor to effective BAN power control, and also gives some background on IEEE standards Tx/Rx power requirements. Section IV gives relevant performance analysis in terms of power savings and reliability of the adapted power control with the predictor. Finally Section V provides some concluding remarks.

\section{EXPERIMENTAL SETUP}

The experimental set-up was for on-body area communications encompassing everyday activity of long periods using multiple small body-mounted radios (channel sounders) as Tx and Rx. The activity is predominantly that of an office-worker over several hours in an indoor office, at home, and jogging in an outdoor suburban environment. The measurements were made at $2360 \mathrm{MHz}$, one of the proposed carrier frequencies for the IEEE 802.15.6 BAN standard [6].

The small wearable radios were placed on 10 adult test subjects, with multiple measurements made for some subjects, at different times. One of the measurement datasets that were used is open-access [7]. A full description of the radios can be found in [8]. Subjects wore a varying number of these radios, with some operating as $\mathrm{Rx}$, some as $\mathrm{Tx}$ and $\mathrm{Rx}$, and 
TABLE I

Channel Sounders Placements; R,L-Right,LeFt. A:AnKle,K:KneE,E:Elbow,H:HiP, W:Wrist

\begin{tabular}{|c|c|c|c|c|c|c|c|c|c|c|}
\hline \multirow[t]{2}{*}{ RECEIVER (Rx) LOCATIONS } & \multicolumn{10}{|c|}{ TRANSMITTER (Tx) LOCATIONS } \\
\hline & \multicolumn{5}{|c|}{ RIGHT HIP } & \multicolumn{5}{|c|}{ LEFT CHEST } \\
\hline FRONT & RA & LA & RK & LK & RW & LW & $\mathrm{RE}$ & LE & LH & HEAD \\
\hline BACK & $\mathrm{RA}$ & LA & RK & LK & RW & LW & $\mathrm{RE}$ & $\mathrm{LE}$ & LH & RH \\
\hline
\end{tabular}

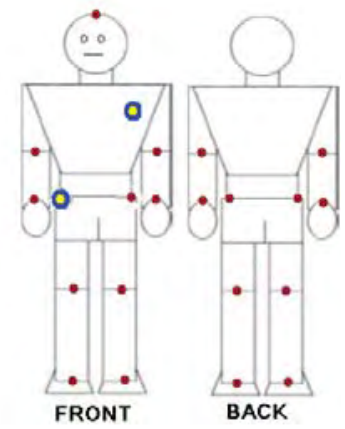

Fig. 1. Full map of Tx/Rx positions for On-Body Measurements

some as $\mathrm{Tx}$ (the total amount of $\mathrm{Tx}$ and $\mathrm{Rx}$ varied from 3 to 20). The complete map of all possible $\mathrm{Tx} / \mathrm{Rx}$ positions is shown in Fig. 1, these are also given in Table I. Individual subject measurements generally lasted for a period of two or more hours. The digital RSSI (Received Signal Strength Indicator) $\log$ made upon successful packet detection by the radios was used to measure channel gain. A total of $140 \mathrm{Tx} / \mathrm{Rx}$ link measurements were made over all test subjects.

\section{Channel-Predictor And POWER Control}

\section{A. IEEE Standards Requirements}

According to the IEEE 802.15.6 draft standard [6], four information Data Rates $\left(R_{D}\right)$ need to be supported for operation in the 2360-2483.5 MHz band; each with associated minimum receive power sensitivities $\left(R x_{\text {sens }}\right)$. These are given in Table II. All standards compliant devices must be able to output Tx power $\left(T x_{\text {out }}\right)$ of at least $-10 \mathrm{dBm}$, and up to a maximum of $0 \mathrm{dBm},[6]$. Knowledge and use of these parameters is vital to testing and operation of effective power control.

TABLE II

BAN COMPLIANT DATA RATES, $R_{D}$, AND ASSOCIATED MINIMUM $R x_{\text {sen }}$ FOR OPERATION AROUND $2.4 \mathrm{GHZ}$

\begin{tabular}{|c|c|c|c|c|}
\hline$R_{D} \mathrm{kbps}$ & 121.4 & 242.9 & 485.7 & 971.4 \\
\hline$R x_{\text {sens }} \mathrm{dBm}$ & -95 & -93 & -90 & -86 \\
\hline
\end{tabular}

BAN devices are required to operate in both $\mathrm{Tx}$ and $\mathrm{Rx}$ mode. Here we describe measures of effective prediction, power control, and reliability in Tx mode (which accounts for just under half of the power consumption in BAN devices [9]).

\section{B. Alternative Weighted Least-Squares Predictor}

The method can first be illustrated in the following diagram Fig. 2. This diagram shows an example portion of a measurement for channel gain RSSI samples (mag) received in a BAN link every $5 \mathrm{~ms}\left(t_{s}=5 \mathrm{~ms}\right)$; which corresponds to the RSSI sampling frequency of the channel sounder. Note that this is somewhat shorter than the relevant BAN superframe periods, but with proper adjustment for much larger sample spacing the method still applies. This portion is partially periodic. In this example, an interval of $N_{s}=1000$ samples (i.e. last $5 \mathrm{~s}$ ) is used. Here we attempt to predict $T_{p r}=30(150 \mathrm{~ms})$ samples ahead of the last received sample by using the 970 channel gain samples that precede it.

The method is to take the last $n_{r}$ received samples and search over the previous $\left(N_{s}-T_{p r}\right)$ samples to find the closest match. (In Fig. 2, $n_{r}=10$; but with a lower sampling rate $N_{s}$ and $n_{r}$ are reduced.) The next $T_{p r}$ samples $\left(\boldsymbol{S}_{\boldsymbol{p}}\right)$ are then predicted by using an alternate least-squares (LS) formulation that is weighted by the last received sample. The predictor can be expressed as follows:

$$
\begin{aligned}
& \boldsymbol{y}_{\boldsymbol{s}}=\boldsymbol{m a g}\left(L-N_{s}+1, L-N_{s}+2, \ldots, L\right), \\
& \boldsymbol{x}(\boldsymbol{i})=\mid \boldsymbol{y}_{\boldsymbol{s}}\left(n_{r}-i+1, \ldots, N_{s}-T_{p r}-i+1\right)- \\
& \left.y_{s}\left(N_{s}-i+1\right)\right|^{2}, i=1, \ldots, n_{r}, \\
& \mathbf{X}_{\mathbf{s}}=\sum_{i=1}^{n_{r}} \boldsymbol{x}(\boldsymbol{i}), \\
& i_{n}=\max \arg \min \left(\mathbf{X}_{\mathbf{s}}\right)+n_{r}, \\
& \boldsymbol{S}_{\boldsymbol{p}}=\left(m_{1} \cdot \boldsymbol{y}_{\boldsymbol{s}}\left(i_{n}, \ldots, i_{n}+T_{p r}-1\right)+\right. \\
& \left.\quad m_{2} \cdot \operatorname{mag}(L)\right) /\left(m_{1}+m_{2}\right),
\end{aligned}
$$

where $|\cdot|$ are the absolute values of the vector difference, $m_{1}=1$, and $m_{2}=2.5$ is the preferred instantiation for BAN

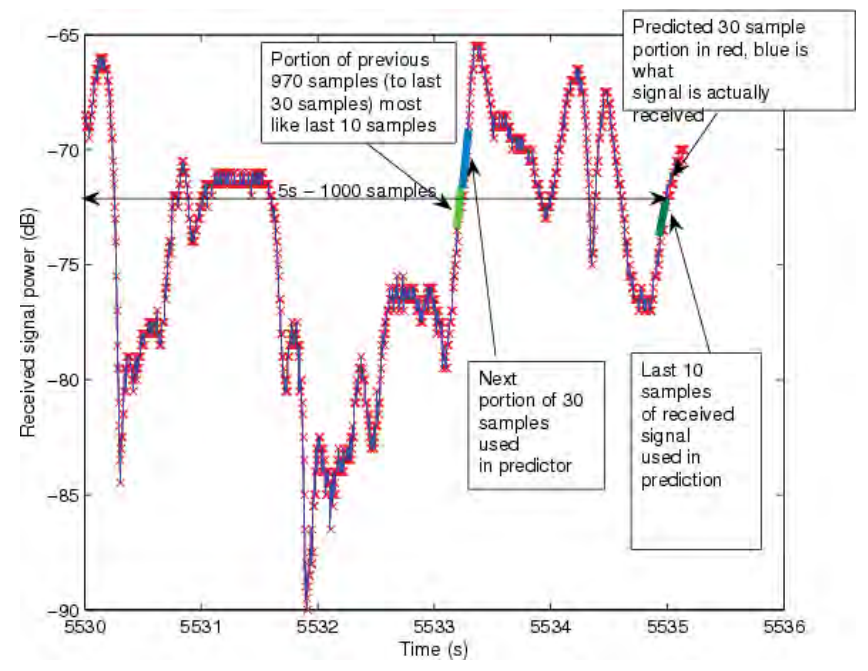

Fig. 2. Illustration measurement with application of channel predictor 
according to a mean-square prediction error criterion. The best instantiation of $N_{s}$ according to the same mean-square prediction error criterion is the number of samples, which changes depending on BAN superframe periods, corresponding to the last $4 \mathrm{~s}$ of signal (i.e., $1 \mathrm{~s}$ less than illustration in Fig. 2). The best values of $n_{r}$ are $n_{r}=5$ for $t_{s}=10 \mathrm{~ms}, n_{r}=4$ for $t_{s}=20 \mathrm{~ms}, n_{r}=3$ for $20<t_{s}<120 \mathrm{~ms}$ and $n_{r}=2$ for $t_{s}>120 \mathrm{~ms} . \boldsymbol{S}_{\boldsymbol{p}}$ is the final predicted channel gain portion, $\operatorname{mag}(L)$ represents the last received channel gain.

If we simply map each predicted channel gain, $S_{p}(\ell)$, to a power estimate, $\hat{p}(\ell)$, (which may not be practical for actual power control as no error margin is allowed), we can directly compare the performance of the predictor described by (1)-(4) to a sample-and-hold method that uses the last received sample, $\operatorname{mag}(L)$, as an estimate of the next $T_{p r}$ samples. A good measure of this is normalized mean square power error (NMSE) and normalized power bias, following from [10], which are given there as $E\left[(\hat{p}(\ell) / p(\ell)-1)^{2}\right]$ and $E[(\hat{p}(\ell) / p(\ell)-1)]$ respectively, where $E[\cdot]$ represents the mean or expectation.

Thus in Fig. 3 we provide the relative NMSE power error of the predictor (1)-(4), and the relative NMSE power error using a held sample as prediction; for prediction intervals up to $2 \mathrm{~s}$, ahead with a receiver sensitivity, $R x_{\text {sens }}$, of $-90 \mathrm{dBm}$, and link sampling periods varying from $10 \mathrm{~ms}$ to $120 \mathrm{~ms}$. The error is based on the NMSE power error of these predictors relative to the NMSE power error of simply transmitting at a power of $-10 \mathrm{dBm}$, which is a typical transmit power for BAN [6]. There is no trend in the relative NMSE predictor power error or the "Hold" sample predictor, hence we do not seek to fit a trend-line, but we note that for all prediction intervals from $120 \mathrm{~ms}$ ahead to $2 \mathrm{~s}$ ahead, there are large improvements shown in Fig. 3, varying from 2-fold to 100-fold improvement of the weighted alternate-least-squares predictor over the "Hold" Predictor at this sensitivity. Fig. 3 also clearly shows good prediction accuracy up to $2 \mathrm{~s}$ ahead, far in excess of the coherence time of $500 \mathrm{~ms}$ for a typical everyday BAN channel. We note the general spread of NMSE power error performance is least for predicting $1000 \mathrm{~ms}$ ahead. In general, many orders of magnitude performance improvement are shown for both the "Hold" method and using the predictor compared to simply transmitting at a power of $-10 \mathrm{dBm}$, which might be expected given the overall median path loss of $71 \mathrm{~dB}$ for all measurements, and large fluctuations in signal strength for each measurement.

For further illustration, we plot the direct ratio of NMSE power error for the predictor to the NMSE power error for the "Hold" Sample predictor, as well as the direct ratio of the normalize power bias of the predictor to that of the "Hold" predictor in Fig. 4 (for a prediction interval 1s ahead, for all receiver sensitivities, $R x_{\text {sens }}$, and link sample spacings, $t_{s}$, varying from $10 \mathrm{~ms}$ to $400 \mathrm{~ms}$ ). Again there is no trend, so we do not attempt to fit a trend-line. As might be expected, bias ratios are larger than NMSE ratios; these bias ratios range from 0.2 to 0.65 , and the NMSE power error ratios vary much more widely from 0.001 to 0.5 . Thus, the proposed prediction method shows significant improvement for the predictor of (1)(4) over using a "held" sample for the wide range of realistic BAN scenarios analysed in Fig. 3 and Fig. 4.

\section{Power Control}

We then adapt the power control scheme used from the sample-and-hold method described in [4] to the predicted signal portion $S_{p}$ derived from (1)-(4). This power control scheme is described in Algorithm 1. The essential scaling of power allocation from estimate $S_{p}$ is designed to meet targeted BAN reliability and power consumption improvements simultaneously. Here here we choose $0.5 \mathrm{~dB}$ steps for power allocation, in contrast to the $2.5 \mathrm{~dB}$ steps in [4] (this gives power savings), and different power allocation based on the short term mean path loss is adapted to slightly change the scaling of power allocation (with more power above the mean, relatively lower power below the mean as for the "Enhanced Hold" Method in

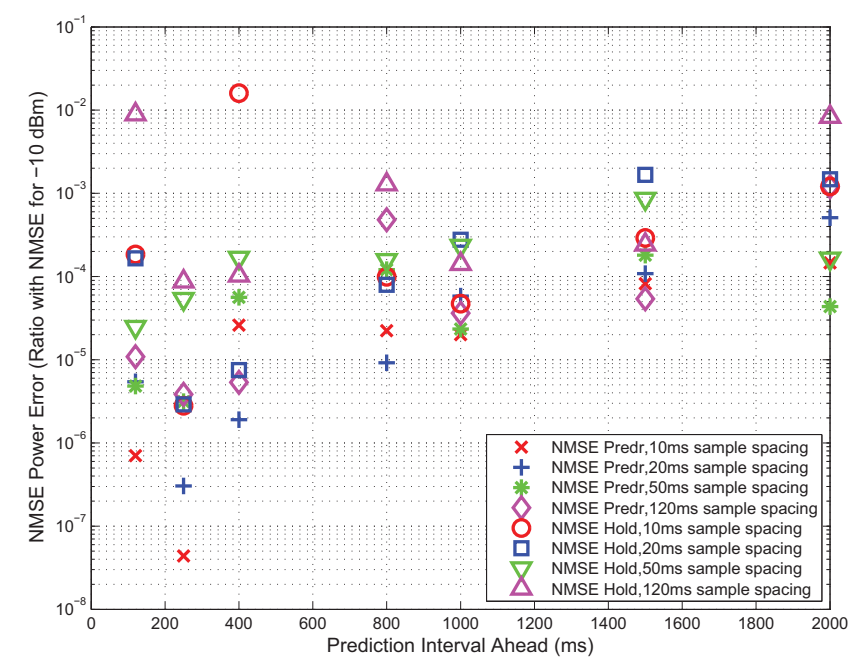

Fig. 3. Normalized Mean Square Power Error of Estimator v. Hold Method, $R x_{\text {sens }}=-90 \mathrm{dBm}$

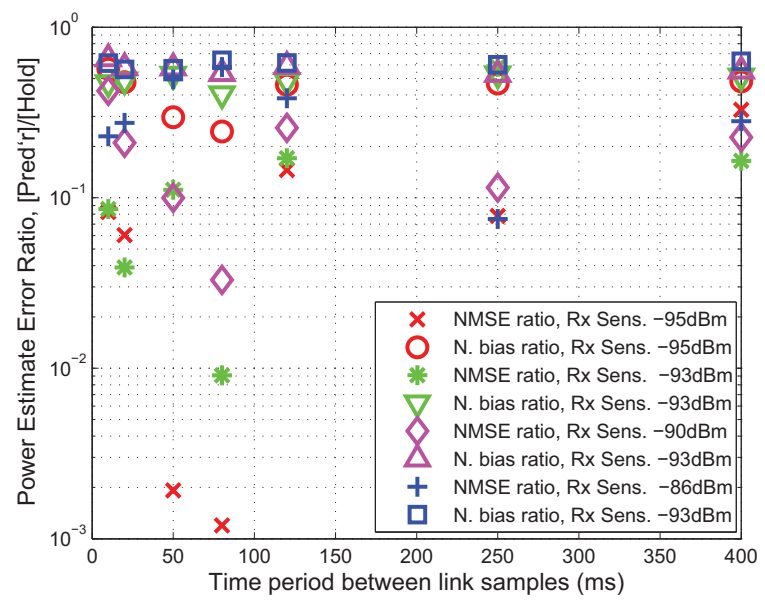

Fig. 4. NMSE power error ratio and Normalized Power Bias Ratio, of proposed predictor to hold predictor, predicting $1 \mathrm{~s}$ ahead 


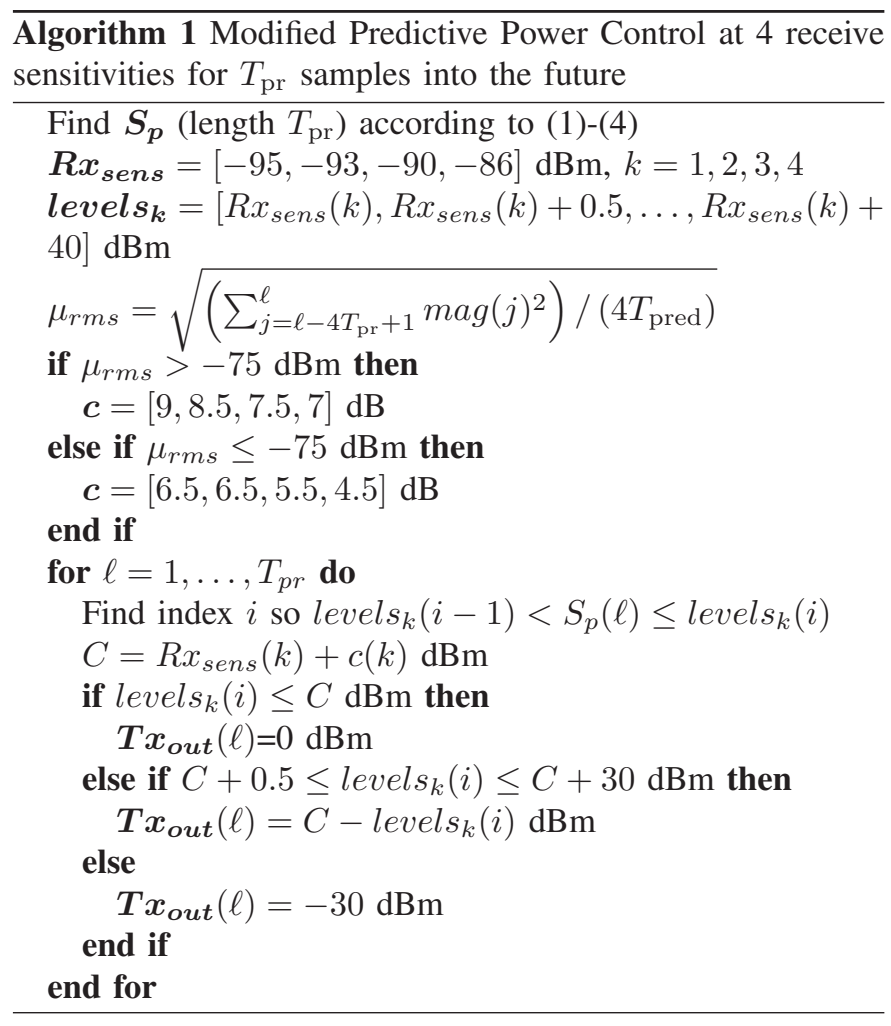

[4]).

\section{Performance Analysis}

Results of extensive testing of comparison of the modified predictor with the "Hold" method and "Enhanced Hold" method of [4] over all channel sounder datasets will now be presented in terms of average power consumption in Tx mode, and percentage outage. Without any explicit $2.4 \mathrm{GHz}$ power consumption data to draw upon, we have used [9] and [11] as a guide to map $T x_{\text {out }}$ to transmit power consumption, $T x_{\text {cons }}$. This was achieved by shifting the $900 \mathrm{MHz}$ power consumption curve in [11] by $2.2 \mathrm{~dB}$ such that it passed through the $\{-10 \mathrm{dBm}, 2.9 \mathrm{~mW}\}$ reference $\left\{T x_{\text {out }}, T x_{\text {cons }}\right\}$ point given in [9] for $2.4 \mathrm{GHz}$ operation. This is of course an approximation, but it will suffice for the purposes of this paper.

In Fig. 5 average circuit power consumption is shown for power control $1 \mathrm{~s}$ ahead, and with sample spacing $t_{s}$ varying from $10 \mathrm{~ms}$ to $400 \mathrm{~ms}$, which are the assumed superframe periods. Both the "Hold" method [4] and the modified predictor applied in Algorithm 1 are used. It is clear from Fig. 5 that the power consumption of each method is relatively constant and independent of the period between link samples. It can be seen in Fig. 5 that with receive sensitivities of $-95,-93$ and $-90 \mathrm{dBm}$, the enhanced hold methods and modified prediction methods both consume less power, between $8 \%$ and $22 \%$ less than transmitting with a constant power of $-10 \mathrm{dBm}$. At a $R x_{\text {sens }}$ of $-86 \mathrm{dBm}$, the circuit power consumption is marginally greater than that at constant $T x_{\text {out }}=-10 \mathrm{dBm}$, and at $-86 \mathrm{dBm}$ there are power savings over constant $T x_{\text {out }}$ at $-7.5 \mathrm{dBm}$. For all schemes there are significant power savings over constant power transmission above $-7.5 \mathrm{dBm}$.

Fig. 6 shows the outage percentage for both power control methods, using predictive power control $400 \mathrm{~ms}$ ahead, and compares them to the "Hold". It is noted that all methods have significantly less than $10 \%$ outage, between $3 \%$ and $5.5 \%$ for all $R x_{\text {sens }}$ less than $-86 \mathrm{dBm}$, and around $8 \%$ for the "Modified Predictor" method at $R x_{\text {sens }}=-86 \mathrm{dBm}$. In general, the "Modified Predictor" method provides a $0.5 \%$ to $1 \%$ improvement over using the "Hold" method. It is noted that these outages provide a lower bound on average packet error rate, which for BAN should be less than $10 \%$ [6]. It can thus be concluded that in most cases for the predictive power control presented, reliable operation according to the draft IEEE BAN standard can be achieved.

In Fig. 7 we present results for outage percentage and average power consumption for the particular case of $\mathrm{Tx} / \mathrm{Rx}$ link sample spacing, $t_{s}=50 \mathrm{~ms}$, for predictive power control

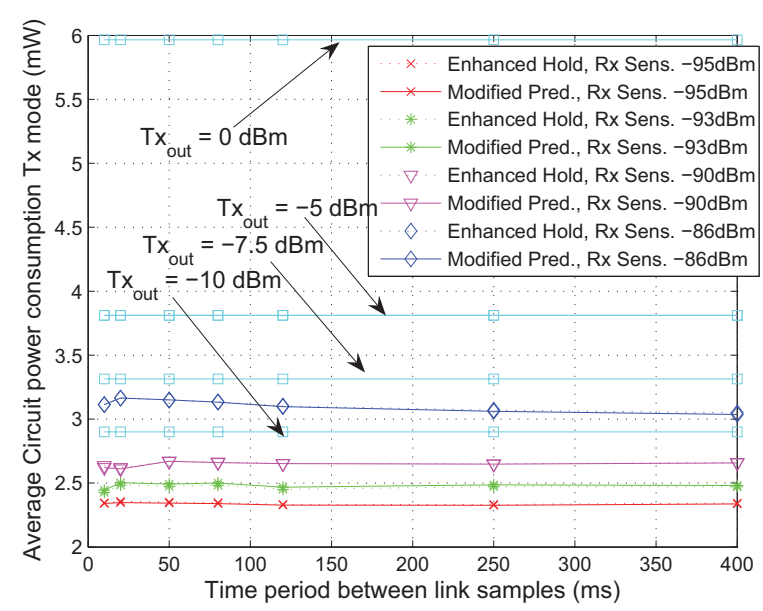

Fig. 5. Transmit (Tx) Mode Power consumption with Modified predictive power control $1000 \mathrm{~ms}$ ahead

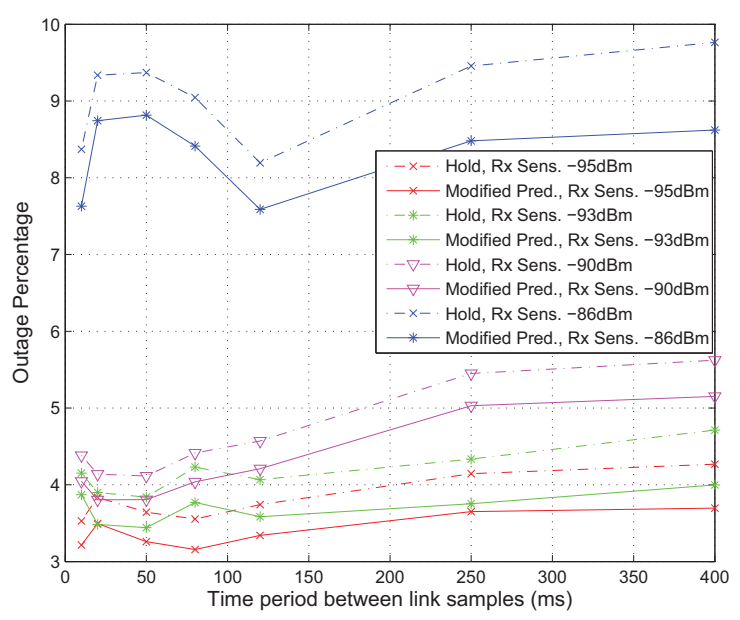

Fig. 6. Percentage Outage, Predictive Power Control $400 \mathrm{~ms}$ ahead 
$250 \mathrm{~ms}, 400 \mathrm{~ms}, 1000 \mathrm{~ms}$, and $2000 \mathrm{~ms}$ ahead. We also provide these results for the "Enhanced Hold" method [4], and results for "Do Nothing", transmitting at constant power of $-10 \mathrm{dBm}$, or greater, as comparison. Outage percentage, Fig. 7(a), increases from around 3\% to around 9\%, which meets the condition for reliable operation in terms of outages. Average power consumption increases in Fig. 7(b) from around $2.4 \mathrm{~mW}$ to around $3.2 \mathrm{~mW}$. Outage percentage for predictive power control is approximate to transmitting at a constant $T x_{\text {out }}=-5 \mathrm{dBm}$ or less. In all but the lowest $R x_{\text {sens }}$, outage percentage for $T x_{\text {out }}=-10 \mathrm{dBm}$ is significantly greater than predictive power control. The outage percentage for constant $T x_{\text {out }}=-10 \mathrm{dBm}$ exceeds $10 \%$ for $R x_{\text {sens }}$ above $-93 \mathrm{dBm}$. Power consumption savings are generally made over all "Do Nothing" cases, with savings always over constant $T x_{\text {out }} \geq-7.5 \mathrm{dBm}$. As the prediction interval increases from $250 \mathrm{~ms}$ to $2 \mathrm{~s}$ outage percentage increases by up to $3 \%$ in Fig. 7 (a) for each $R x_{\text {sens }}$. Power consumption varies by up to $0.35 \mathrm{~mW}$ in Fig. 7(b), with the least sensitive receiver. Importantly, there is only very minor improvement in terms of the reliability of predictive power control over the "Enhanced Hold" method shown in Fig. 7(a), where there is almost identical power consumption over the range of receive sensitivities.

\section{CONCLUding REMARKS}

A novel long-term channel predictor particularly suited to the on-body area communications channel has been presented. It utilizes the partial periodicity of the BAN channel and weights an alternate least-squares estimate for the desired prediction interval using the last $4 \mathrm{~s}$ of received signal. As a predictor it performs significantly better than using a single held sample for prediction. When mapped to a suitable power allocation scheme for proper BAN operation, there are concurrent improvements in reliability and power consumption in comparison to some typical BAN transmission strategies with no channel prediction. When applied to sensible power control, it generally performs a little better than using a held sample alone, but only gives very minor improvement over the enhanced method where the "held" sample is based on a short-term mean path loss of the BAN channel.

\section{REFERENCES}

[1] D. Lewis, "802.15.6 call for applications - response summary," July 2008, IEEE802.15.6 technical contribution, document ID:15-08-040705 .

[2] B. Zhen, M. Patel, S. Lee, and E. Won, "Body Area Network (BAN) Technical Requirements," July 2008, IEEE802.15.6 technical contribution, document ID:15-08-0037-01-0006-ieee-802-15-6technical-requirements-document-v-4-0.

[3] D. Smith, L. Hanlen, J. Zhang, D. Miniutti, D. Rodda, and B. Gilbert, "First- and second-order statistical characterizations of the dynamic body area propagation channel of various bandwidths," Annals of Telecommunications, vol. 66, pp. 187-203, 2011, 10.1007/s12243-010-0233-8. [Online]. Available: http://dx.doi.org/10.1007/s12243-010-0233-8

[4] D. Smith, T. Lamahewa, L. Hanlen, and D. Miniutti, "Simple predictionbased power control for the on-body area communications channel," in Communications (ICC), 2011 IEEE International Conference on, june 2011, pp. $1-5$.

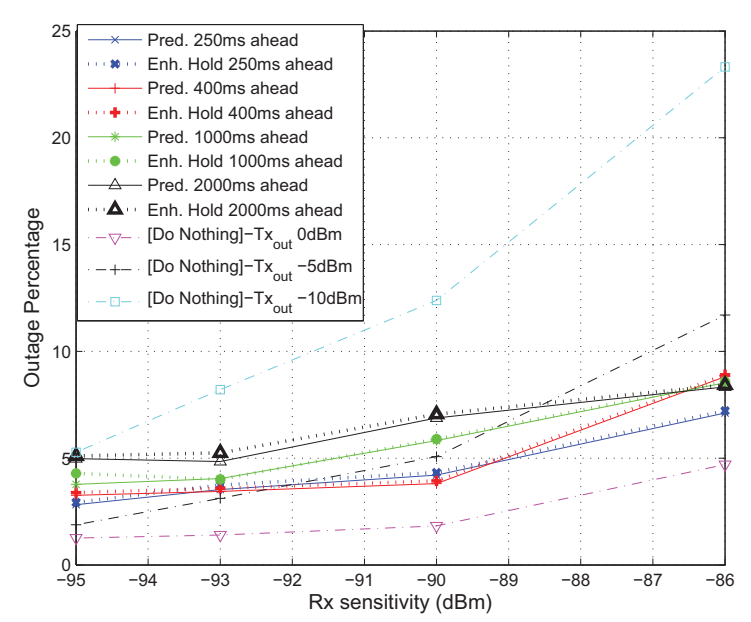

(a) Outage Percentage

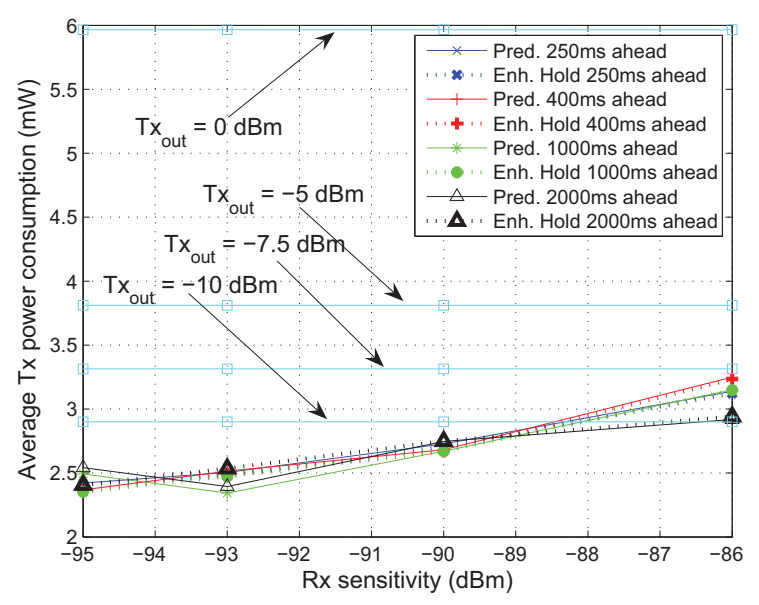

(b) Average Tx power consumption

Fig. 7. Transmit (Tx) Mode Outage and Power consumption, $50 \mathrm{~ms}$ link sample spacing, Power control using Modified Predictor, $250 \mathrm{~ms}$ to $2 \mathrm{~s}$ ahead, in terms of $R x_{\text {sens }}$

[5] R. Kazemi, R. Vesilo, E. Dutkiewicz, and R. Liu, "Dynamic power control in wireless body area networks using reinforcement learning with approximation," in 2011 IEEE International Symposium on Personal, Indoor and Mobile Radio Communications, PIMRC, Toronto, Canada, Sep. 2011

[6] A. Astrin et al., "Tg6 draft," May 2010, IEEE802.15.6 technical contribution, document ID:15-10-0245-040245-06-0006-tg6-draft.

[7] L. W. Hanlen and D. Rodda, "Public dataset for BAN radio channel models," online www.nicta.com.au/hpi, March 2010.

[8] L. Hanlen et al., "Open-source testbed for body area networks: 200 sample/sec, 12 hrs continuous measurement." in Personal Indoor and Mobile Radio Communication, 2010 IEEE 20th International Symposium on, Istanbul,Turkey, Sep. 2010, pp. 1-5.

[9] D. Davenport et al., "Medwin physical layer proposal," May 2009 , IEEE802.15.6 technical contribution, document ID:15-09-0328-010006-medwin-physical-layer-proposal-presentation.

[10] R. Narasimhan and D. Cox, "Estimation of mobile speed and average received power in wireless systems using best basis methods," Cотmunications, IEEE Transactions on, vol. 49, no. 12, pp. $2172-2183$, Dec. 2001.

[11] D. Sagan, “Ultra-low power medical BAN PHY proposal,” May 2009, IEEE802.15.6 ID:15-09-0351-00-0006-ulp-medical-ban-phy-proposal. 